Article

\title{
The Chemical Composition, Antioxidant and Anti-Tyrosinase Activities, and Irritation Properties of Sripanya Punica granatum Peel Extract
}

\author{
Nachtharinee Laosirisathian ${ }^{1,2}$, Chalermpong Saenjum ${ }^{2}$, Jakkapan Sirithunyalug ${ }^{2,3}$, \\ Sukum Eitssayeam ${ }^{4}$, Busaban Sirithunyalug ${ }^{2, *}$ and Wantida Chaiyana $2,3, * \mathbb{D}$ \\ 1 Graduate School, Chiang Mai University, Chiang Mai 50200, Thailand; nachtharineelao@gmail.com \\ 2 Department of Pharmaceutical Science, Faculty of Pharmacy, Chiang Mai University, \\ Chiang Mai 50200, Thailand; chalermpong.saenjum@gmail.com (C.S.); jakkapan.s@cmu.ac.th (J.S.) \\ 3 Research Center of Pharmaceutical Nanotechnology, Chiang Mai University, Chiang Mai 50200, Thailand \\ 4 Department of Physics and Materials Science, Faculty of Science, Chiang Mai University, \\ Chiang Mai 50200, Thailand; sukum99@yahoo.com \\ * Correspondence: busaban.s@cmu.ac.th (B.S.); wantida.chaiyana@gmail.com (W.C.); \\ Tel.: +66-5394-4343 (B.S. \& W.C.)
}

Received: 16 December 2019; Accepted: 13 January 2020; Published: 14 January 2020

\begin{abstract}
This is the first study to investigate the effect of ethanol concentrations on the phenolic content and biological activities of Sripanya Punica granatum peel extract. Various concentrations of ethanol, (20 to $80 \% \mathrm{v} / \mathrm{v}$ ), were used in the maceration process. Extracts were investigated for total phenolic content by the Folin-Ciocalteu method, antioxidant activities by 1,1-diphenyl-2-picrylhydrazyl (DPPH) and 2,2'-azinobis(3-ethylbenzothiazoline-6-sulfonic acid) (ABTS) assay, and anti-tyrosinase activity by a spectrophotometric method. The chemical composition was investigated using high-performance liquid chromatography. The irritation of the extracts was determined by Hen's egg test-chorioallantoic membrane assay. The results demonstrated that E60, which was extracted using $60 \% \mathrm{v} / \mathrm{v}$ ethanol, had the highest yield $(66.23 \pm 0.38 \% \mathrm{w} / \mathrm{w})$, the highest total phenolic content $(490.42 \pm 7.20 \mu \mathrm{g}$ GAE/g), and the highest antioxidant and anti-tyrosinase activities. $\mathrm{IC}_{50}$ values on $\mathrm{DPPH}^{\bullet}$ and ABTS $^{\bullet+}$ inhibition were $10.97 \pm 0.97$ and $6.48 \pm 0.06 \mu \mathrm{g} / \mathrm{mL}$, respectively. The $60 \% \mathrm{v} / \mathrm{v}$ ethanol extract (E60) possessed strong tyrosinase inhibition with an $\mathrm{IC}_{50}$ value of $0.10 \pm 0.13 \mu \mathrm{g} / \mathrm{mL}$, which is more potent than kojic acid. The major constituents were punicalagin $(4.05 \pm 0.26 \% \mathrm{w} / \mathrm{w})$ and ellagic acid $(0.63 \pm 0.04 \% \mathrm{w} / \mathrm{w})$. E60 was safe for topical application since it did not induce irritation. Therefore, we suggest that E60 is a promising agent for further product development.
\end{abstract}

Keywords: Sripanya; Punica granatum; antioxidant; anti-tyrosinase; irritation; HET-CAM

\section{Introduction}

Punica granatum, a medicinal plant in the family of Punicaceae, is known as pomegranate or punica apple [1]. The pomegranate is one of the most popular fruits native to Iran. It is widely grown in several tropical and subtropical countries, such as Afghanistan, Turkey, Egypt, Italy, India, China, Thailand, Chile, Spain, and the United States [2,3]. However, numerous pomegranate varieties have not yet been reported.

In Thailand, four pomegranate varieties, including Dentawan, Jarassang, Pechamporn, and Sripanya, have been discovered and registered by the Plant Varieties Protection Office of the Department of Agricultural Extension. These varieties have several identifiable characteristics compared to other existing varieties, such as the peel color (ranging from yellow to purple, pink and red, with the latter being the most common), seed-coat color (ranging from white to red), seed hardness, 
juice content, sweetness, and astringency. The Dentawan and Pechamporn varieties were imported from India and can provide a good yield throughout the year in the Nakhon Pathom province in Thailand. They differ in peel color and seed-coat color. Dentawan has a red peel and red seed coat, whereas Pechamporn has an orange-red peel and white-pink seed coat. Although both of them are soft and sweet, they are not popular among consumers because Dentawan can only be grown in the Nakhon Pathom, and Pechamporn has a very thick peel.

On the other hand, Jarassang, bred from an Indian and Thai native variety, can provide good yields in May, August, and December in the Nakhon Ratchasima province in Thailand. Jarassang has a red-yellow peel and orange-red seed coat. Sripanya, which is the most popular pomegranate in Thailand, has a red peel, a dark-red seed coat, and a very sweet taste. It was bred from a Spanish and Thai native variety. It can provide a good yield throughout the year in the Tak province. The Phop Phra district is situated 260-1700 m above sea level and is near the Earth's equator, according to the District Office of Phop Phra. Additionally, Sripanya, an economic plant, is used in commercial pomegranate-juice production as it supplies a plentiful amount of juice. These manufacturing procedures generate large quantities of Sripanya pomegranate peel as waste products.

Various parts of the pomegranate tree, including the tree bark, fruit peel, seeds, and juice, have been used extensively as rejuvenation remedies since ancient times for the treatment of diarrhea, dysentery, intestinal parasites, gum bleeding, nose bleeds, and hemorrhoids. Additionally, several biological activities of the pomegranate peel have also been investigated, including antioxidant, anticarcinogenic, anti-inflammatory, anti-cardiovascular, antidiabetic, and antimicrobial activities. The medicinal value of the pomegranate peel is due to its main constituents of tannins, polyphenolic compounds, ellagictannin, and ellagic acid, which possess antioxidant, anti-tyrosinase, and anti-inflammatory activities [1,4-6]. Moreover, pomegranates have been used for cosmetic purposes as they can effectively reduce skin pigmentation in guinea pigs [7].

There have hitherto been no previous reports concerning Sripanya pomegranate peel. This study is the first to investigate suitable extraction solvents for the bioactive compounds extracted from Sripanya pomegranate peel. Moreover, the antioxidant and anti-tyrosinase activities, as well as the major chemical composition of Sripanya pomegranate peel extracts, were investigated. The irritation effect of the extracts are also reported.

\section{Materials and Methods}

\subsection{Plant Material}

The waste of Sripanya pomegranate peel was collected from the Phop Phra district of Tak, Thailand, from September to November 2017, with a voucher specimen number SPES-001. The peel was separated from the seeds before being washed and dried in a hot-air oven at $60^{\circ} \mathrm{C}$ for $48 \mathrm{~h}$ and ground into powder using a vertical roller mill (Conversant Technology, Co., Ltd., Bangkok, Thailand). The dried powder of the Sripanya pomegranate peel was then sieved through an 80-mesh sieve and kept in a sealed container at room temperature until use. The moisture content, and loss upon drying, of dried Sripanya pomegranate peel powder were evaluated using a digital moisture analyzer (MX-50, A \& D Company, Ltd., Tokyo, Japan). About 2-6 g of the dried powder was accurately weighed and transferred to an aluminum pan and held at $105^{\circ} \mathrm{C}$ until the sample weight was constant.

\subsection{Chemical Reagents}

The analytical grade reagents including absolute ethanol, Folin-Ciocalteu reagent, potassium persulfate, and hydrochloric acid were purchased from Merck, Darmstadt, Germany; 1,1-diphenyl-2-picrylhydrazyl (DPPH) and 2,2'-azino-bis (3-ethylbenzthiazoline-6-sulfonic acid) (ABTS) were purchased from Sigma-Aldrich, Steinheim, Switzerland; dihydrochloride mushroom tyrosinase was purchased from Sigma-Aldrich, St. Louis, MO, USA; L-tyrosine was purchased from Sigma-Aldrich, Darmstadt, Germany; sodium carbonate was purchased from Ajax Finechem Pty., Ltd., Taren Point, 
Australia; dimethyl sulfoxide, acetonitrile, sodium hydroxide, and deionized water were purchased from RCI Labscan, Ltd., Bangkok, Thailand; and glacial acetic acid was purchased from Fisher Scientific, Loughborough, UK. The standard substances-gallic acid, ellagic acid, punicalagin, kojic acid and trolox-were purchased from Sigma-Aldrich, Buchs, Switzerland. Tween 20 and sodium lauryl sulfate were purchased from Hong Huat, Bangkok, Thailand.

\subsection{Preparation of Sripanya Pomegranate Peel Extract}

The dried powder of Sripanya pomegranate peel was extracted using a maceration method in various concentrations of ethanol, including $20 \%, 30 \%, 40 \%, 50 \%, 60 \%, 70 \%$, and $80 \% \mathrm{v} / \mathrm{v}$. Briefly, $15 \mathrm{~g}$ of the dried Sripanya pomegranate peel powder were macerated in $500 \mathrm{~mL}$ of each solvent and continuously stirred using a magnetic stirrer at $5000 \mathrm{rpm}$, at $50^{\circ} \mathrm{C}$, for $15 \mathrm{~min}$ before being filtered through Whatman no. 1 filter paper. The solvent was then removed by the rotary evaporator (Tokyo Rikakikai, Co., Ltd., Tokyo, Japan) with the water bath temperature set at $50{ }^{\circ} \mathrm{C}$ under vacuum. E20, E30, E40, E50, E60, E70, and E80 were obtained from the maceration using 20\%, 30\%, 40\%, 50\%, $60 \%, 70 \%$, and $80 \% \mathrm{v} / \mathrm{v}$ of ethanol, respectively. The extract was kept in a sealed container at $4{ }^{\circ} \mathrm{C}$ until used. The extractions were done in triplicate. The yield of each extract was calculated using the following equation:

$$
\text { Yield }(\%)=(\mathrm{W} 1 \times 100) /(\mathrm{W} 2)
$$

where W1 is the weight of the dried Sripanya pomegranate peel extract, and W2 is the weight of the dried Sripanya pomegranate peel powder.

\subsection{Total Phenolic Content Determination}

Total phenolic concentrations of Sripanya pomegranate peel extracts were determined by the Folin-Ciocalteu (FC) colorimetric method according to the method of Li et al. [8] with some modifications. Briefly, $100 \mu \mathrm{L}$ of 1:10 diluted Folin-Ciocalteu reagent was mixed with $20 \mu \mathrm{L}$ of the test sample solution and incubated for $4 \mathrm{~min}$ at room temperature. After that, $80 \mu \mathrm{L}$ of a saturated sodium carbonate solution $(0.75 \mathrm{~g} / \mathrm{mL})$ was added to the mixture and incubated for another $2 \mathrm{~h}$ at room temperature. The absorbance of the mixtures was measured at $760 \mathrm{~nm}$ using a microplate reader (Beckman Coulter, Inc., Lagerhausstrasse, Austria). All experiments were performed in triplicate, and gallic acid was used as a standard substance.

\subsection{Antioxidant Activity Determination}

\subsubsection{1,1-Diphenyl-2-Picrylhydrazyl Radical Scavenging (DPPH) Assay}

The scavenging activity on the DPPH ${ }^{\bullet}$ of Sripanya pomegranate peel extracts was determined via DPPH assay according to the method of Brem et al. [9], with some modifications. Briefly, $180 \mu \mathrm{L}$ of the $\mathrm{DPPH}^{\bullet}$ solution were mixed with $20 \mu \mathrm{L}$ of the test sample solution in DMSO and incubated in the dark for $30 \mathrm{~min}$. The absorbance of the mixture was measured at $520 \mathrm{~nm}$ using a microplate reader (Spectrostar Nano, BMG Labtech $\mathrm{GmbH}$, Ortenberg, Germany). The scavenging activities on the $\mathrm{DPPH}^{\bullet}$ of the Sripanya pomegranate peel extracts were reported as \% inhibition, which was calculated using the following equation:

$$
\% \text { inhibition }=[(\mathrm{A}-\mathrm{B})-(\mathrm{C}-\mathrm{D}) /(\mathrm{A}-\mathrm{B})] \times 100
$$

where A is the absorbance of DMSO and the DPPH solution; B is the absorbance of DMSO and DI water; $C$ is the absorbance of the sample and DPPH solution; and D is the absorbance of the sample and DI water. $\mathrm{IC}_{50}$ was calculated using GraphPad Prism version 2.01. All experiments were performed in triplicate, and gallic acid was used as a standard substance. 


\subsubsection{2,2'-Azinobis(3-Ethylbenzothiazoline-6-Sulphonic Acid) (ABTS•) Radical Scavenging Assay}

The ABTS ${ }^{\bullet+}$ scavenging activity of Sripanya pomegranate peel extracts were determined by ABTS assay according to the method of Tachakittirungrod et al. [10], with some modifications. Briefly, $2 \mathrm{~mL}$ of the $7 \mathrm{mM}$ ABTS solution was mixed with $3 \mathrm{~mL}$, or $2.45 \mathrm{mM}$, of potassium persulfate solution to generate the $\mathrm{ABTS}^{\bullet+}$ solution. After incubation in the dark for $24 \mathrm{~h}$, the ABTS ${ }^{\bullet+}$ solution was diluted 20 times by ethanol to obtain an absorbance of $0.7 \pm 0.1$ units at $750 \mathrm{~nm}$. Afterward, $180 \mu \mathrm{L}$ of the ABTS $^{\bullet+}$ solution was mixed with $20 \mu \mathrm{L}$ of the test sample solution in DMSO. After $5 \mathrm{~min}$, the absorbance of the mixture was measured at $750 \mathrm{~nm}$ using a microplate reader (Spectrostar Nano, BMG Labtech GmbH, Ortenberg, Germany). The ABTS ${ }^{\bullet+}$ scavenging activity of Sripanya pomegranate peel extracts were reported as \% inhibition, which was calculated using the following equation:

$$
\% \text { inhibition }=[(\mathrm{A}-\mathrm{B})-(\mathrm{C}-\mathrm{D}) /(\mathrm{A}-\mathrm{B})] \times 100
$$

where A is the absorbance of DMSO and the ABTS solution; $\mathrm{B}$ is the absorbance of DMSO and DI water; $C$ is the absorbance of the sample and the ABTS solution; and D is the absorbance of the sample and DI water. $\mathrm{IC}_{50}$ was calculated using GraphPad Prism version 2.01. All experiments were performed in triplicate, and trolox was used as a standard substance.

\subsection{Tyrosinase Inhibitory Activity Determination}

The tyrosinase inhibitory activity of Sripanya pomegranate peel extracts was determined via a tyrosinase inhibitory assay according to the method of Saeio et al. [11], with some modifications. Briefly, $10 \mu \mathrm{L}$ of the test sample solution was mixed with $30 \mu \mathrm{L}$ or 250 units $/ \mathrm{mL}$ of the tyrosinase enzyme and $60 \mu \mathrm{L}$ of PBS with a pH of 6.8. After incubation at room temperature for $10 \mathrm{~min}, 100 \mu \mathrm{L}$ of $2.5 \mathrm{mM}$ L-tyrosine was added and incubated again for $30 \mathrm{~min}$. The absorbance of the mixture was measured with a wavelength of $450 \mathrm{~nm}$ using a microplate reader (Spectrostar Nano, BMG Labtech $\mathrm{GmbH}$, Ortenberg, Germany). The tyrosinase inhibitory activity of Sripanya pomegranate peel extracts was reported as \% inhibition, which was calculated using the following equation:

$$
\% \text { inhibition }=[(\mathrm{A}-\mathrm{B})-(\mathrm{C}-\mathrm{D}) /(\mathrm{A}-\mathrm{B})] \times 100
$$

where $\mathrm{A}$ is the absorbance of ethanol, 250 units/mL of the tyrosinase enzyme, and L-tyrosine; $\mathrm{B}$ is the absorbance of ethanol, 250 units/mL of the tyrosinase enzyme, and PBS with a pH of 6.8; C is the absorbance of the sample, 250 units $/ \mathrm{mL}$ of the tyrosinase enzyme, and L-tyrosine; and $\mathrm{D}$ is the absorbance of the sample, 250 units/mL of the tyrosinase enzyme, and PBS with a pH of 6.8. $\mathrm{IC}_{50}$ was calculated using GraphPad Prism version 2.01. All experiments were performed in triplicate, and kojic acid was used as a standard substance.

\subsection{High-Performance Liquid Chromatography (HPLC) Analysis}

HPLC analysis was used to investigate the chemical compositions of Sripanya pomegranate peel extracts. The HPLC method was modified from the previous study of Li et al. [12]. The Agilent 1100 series equipped with the corresponding photodiode array detector (Hewlett Packard, Waldbronn, Germany), a column-temperature controller, and an auto sampler. Data analysis was performed with the Agilent 3D ChemStation software (Hewlett Packard, Waldbronn, Germany). The chromatographic separation was performed on a phenomenex C-18 column $(5 \mu \mathrm{m}, 4.6 \times 250 \mathrm{~mm})$ using a glacial acetic acid solution with a pH of 3.0 (A) and acetonitrile (B) as a mobile phase. The mobile phase was set at a flow rate of $1 \mathrm{~mL} / \mathrm{min}$. The gradient program was set as follows: $0-10 \mathrm{~min}, 5-10 \% \mathrm{~B}$; $10-30 \mathrm{~min}, 10-15 \% \mathrm{~B}$; and $30-40 \mathrm{~min}, 15-20 \% \mathrm{~B}$. The column temperature was maintained at $30{ }^{\circ} \mathrm{C}$. The chromatogram was detected by a UV-spectrophotometer (Hewlett Packard, Waldbronn, Germany) at a wavelength of $280 \mathrm{~nm}$. The injection volume of each sample and standard solutions was $10 \mu \mathrm{L}$. The HPLC mobile phase, samples, and standard solutions were filtered through a $0.45 \mu \mathrm{m}$ membrane 
filter (ANPEL Laboratory Technologies, Inc., Shanghai, China) before use. The samples were dissolved in HPLC-grade methanol at a concentration of $0.25 \mathrm{mg} / \mathrm{mL}$. Punicalagin and ellagic acid were used as standard compounds in the HPLC analysis.

The punicalagin A content was calculated using the following equation:

$$
x=(y+3297.2) / 15.611,\left(\mathrm{R}^{2}\right)=0.9997
$$

where $x$ is the concentration of punicalagin $A(\mathrm{mg} / \mathrm{L})$, and $y$ is the area under the curve (AUC) of the HPLC chromatogram peak of punicalagin A at a retention time of around $16.5 \mathrm{~min}(\mathrm{mAU})$.

The punicalagin B content was calculated using the following equation:

$$
x=(y+3623.3) / 17.624,\left(\mathrm{R}^{2}\right)=0.9996
$$

where $x$ is the concentration of punicalagin $B(\mathrm{mg} / \mathrm{L})$, and $y$ is the area under the curve (AUC) of the HPLC chromatogram peak of punicalagin B at a retention time of around $17.8 \mathrm{~min}$ (mAU).

The ellagic acid content was calculated using the following equation:

$$
\mathrm{x}=(\mathrm{y}+1446) / 43.511,\left(\mathrm{R}^{2}\right)=0.9992
$$

where $x$ is the concentration of ellagic acid $(\mathrm{mg} / \mathrm{L})$, and $y$ is the area under the curve (AUC) of the HPLC chromatogram peak of ellagic acid, at a retention time of around $28.9 \mathrm{~min}(\mathrm{mAU})$.

\subsection{Irritation Test by Hen's Egg Test-Chorioallantoic Membrane (HET-CAM) Assay}

An acute irritation test of Sripanya pomegranate peel extracts was determined by a modified HET-CAM method [13,14]. The fertilized eggs were obtained from the Faculty of Agriculture, Chiang Mai University. The eggs were kept for 10 days in an incubator set at $37 \pm 1{ }^{\circ} \mathrm{C}$ and $55 \pm 0.5 \% \mathrm{RH}$. After that, the eggshell was opened using a dental rotary saw-blade. After removing the white egg membrane, viable embryos with the yolk sac were kept in the incubator. The samples were then placed on the chorioallantoic membrane (CAM). The irritation reactions including vascular lysis, vascular hemorrhages, and vascular coagulation, were observed within $300 \mathrm{~s}$ after sample applications via a stereo microscope (OLYMPUS, Spacemed, Bangkok, Thailand). Blood vessels on the CAM were continually investigated for $1 \mathrm{~h}$ for long-term irritation. The photographs were taken under a stereo microscope (OLYMPUS, Spacemed, Bangkok, Thailand). The irritation score (IS) was calculated using the following equation:

$$
\mathrm{IS}=[(301-\mathrm{t}(\mathrm{h}) / 300 \times 5]+[(301-\mathrm{t}(1) / 300 \times 7]+[(301-\mathrm{t}(\mathrm{c}) / 300 \times 9]
$$

where $t(h)$ is the time of the first vascular hemorrhages, $t(l)$ is the time of the first vascular lysis, and $t(c)$ is the time of the first vascular coagulation. The time was recorded in seconds. Result classification was as follows: no irritation: IS $=0-0.9$; slight irritation: IS $=1.0-4.9$; moderate irritation: IS $=5.0-8.9$; severe irritation: IS =9.0-21.0. All experiments were performed in duplicate. Sodium lauryl sulfate $(1 \% \mathrm{w} / \mathrm{w})$ was used as a positive control, and normal saline solution $(0.9 \% \mathrm{w} / \mathrm{w} \mathrm{NaCl})$ was used as a negative control.

\subsection{Statistical Analysis}

The mean values of the results were calculated for all triplicate experiments, and the results were expressed as mean \pm standard deviation. The data was subjected to a $t$-test and one-way ANOVA, with a statistical difference of $p<0.05$. 


\section{Results and Discussion}

\subsection{Dried Sripanya Pomegranate Peel Powder}

The dried powder of Sripanya pomegranate peel was fine and yellow. The average moisture content and loss upon drying of the powder were $8.04 \pm 0.06 \%$ and $7.55 \pm 0.10 \%$, respectively. The results were in an acceptance citeria of $\mathrm{WHO}$, who designate an average moisture content and loss upon the drying of the plant materials as no more than $10 \%$ [5]. Therefore, the raw material of the Sripanya pomegranate peel met the quality standard for further use.

\subsection{The Effect of Ethanol Concentration on the Yield of Sripanya Pomegranate Peel Extracts}

All Sripanya pomegranate peel extracts were dark-brown semisolid masses. The yields of each extract are shown in Figure 1. The results show that a higher concentration of ethanol led to a higher yield of Sripanya pomegranate peel extracts. A likely explanation is that the chemical components of the Sripanya pomegranate peels were able to dissolve better in ethanol $(\varepsilon=24.5)$ than DI water $(\varepsilon=80.1)$. However, the maximum yield was obtained at an ethanol concentration of $60 \% \mathrm{v} / \mathrm{v}(p<0.05)$. The yield then tended to decrease when the ethanol concentraion was $70 \%$ and over. This might be because the maximum extraction efficiency of ethanol had already been reached at $60 \% \mathrm{v} / \mathrm{v}$ and no more semipolar compounds could be extracted by higher concentrations of ethanol. Lower polar compounds could have been extracted less due to the lower content of DI water in the extracted solvents. Therefore, it should be concluded that the concentration of ethanol that is most suitable for the highest yield $(66.23 \pm 0.38 \% \mathrm{w} / \mathrm{w})$ of Sripanya pomegranate peel extracts is $60 \% \mathrm{v} / \mathrm{v}(p<0.05)$.

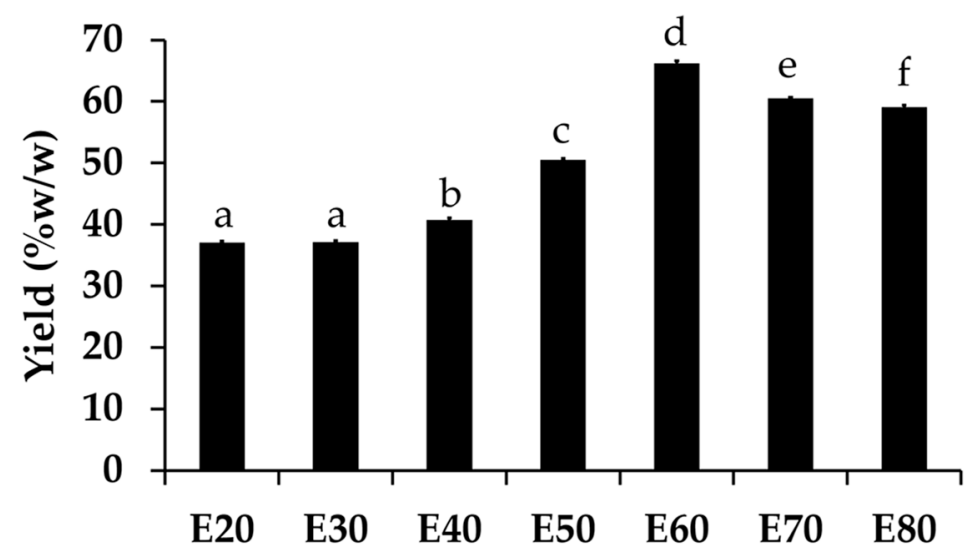

Figure 1. Yield of Sripanya pomegranate peel extracts. E20, E30, E40, E50, E60, E70, and E80 were Sripanya pomegranate peel extracts obtained by maceration in $20 \%, 30 \%, 40 \%, 50 \%, 60 \%, 70 \%$, and $80 \%$ $\mathrm{v} / \mathrm{v}$ of ethanol, respectively. Different letters denote statistically significant differences among groups; $p<0.05)$.

\subsection{Total Phenolic Content of Sripanya Pomegranate Peel Extracts}

Total phenolic concentrations of Sripanya pomegranate peel extracts are shown in Figure 2. The results interestingly show that a $60 \% \mathrm{v} / \mathrm{v}$ or higher concentration of ethanol yielded the highest total phenolic concentrations $(p<0.05)$, and a plateau of total phenolic content was observed. Ethanol concentrations of $60 \%, 70 \%$, and $80 \% \mathrm{v} / \mathrm{v}$ yielded the same concentrations of phenolic compounds $(p>0.05)$, which were $490.42 \pm 7.20,490.12 \pm 3.65$, and $490.07 \pm 2.02 \mu \mathrm{g} \mathrm{GAE} / \mathrm{g}$, respectively. 


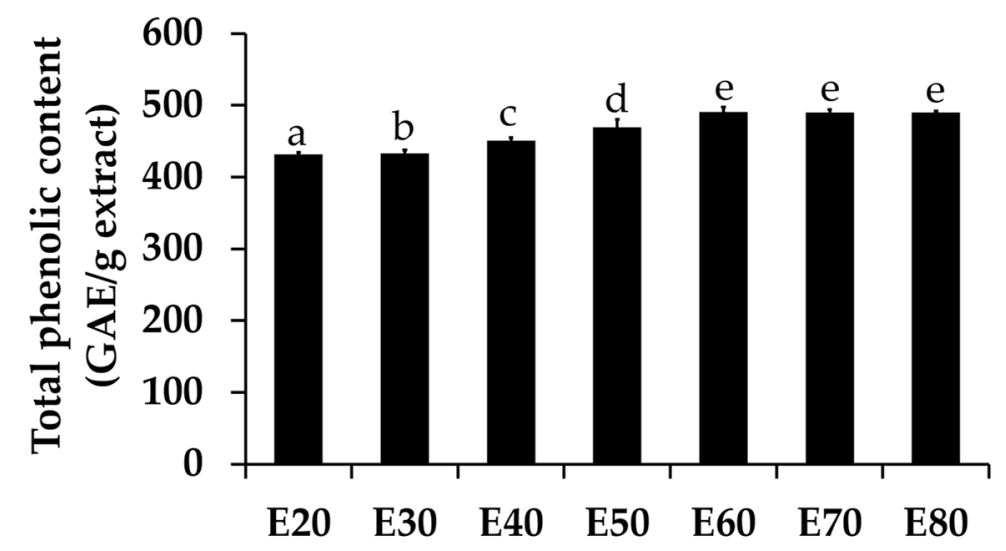

Figure 2. Total phenolic concentrations of Sripanya pomegranate peel extracts. E20, E30, E40, E50, E60, E70, and E80 were Sripanya pomegranate peel extracts obtained by maceration in $20 \%, 30 \%, 40 \%, 50 \%$, $60 \%, 70 \%$, and $80 \% \mathrm{v} / \mathrm{v}$ of ethanol. Different letters denote statistically significant differences among groups; $p<0.05$.

Similar to the above results of the extracted yields, it can be seen that phenolic compounds were extracted better using ethanol than DI water. Higher concentrations of ethanol in the extraction solvents led to higher total phenolic concentrations. These results are in good agreement with previous studies reporting that phenolic compounds, such as anthocyanins, hydroxycinnamic acids, and hydroxybenzoic acids, were more soluble in organic solvents compared to DI water [1,6]. Interstingly, the maximum yield and maximum total phenolic concentrations were obtained at the same concentration of ethanol in the extract solvents, which was $60 \% \mathrm{v} / \mathrm{v}$. Therefore, it might be concluded that the most abundant components of Sripanya pomegranate peel extracts are phenolic compounds, which can be extracted well by ethanol, the most effective concentration of which is $60 \% \mathrm{v} / \mathrm{v}$. Several previous studies have also confirmed that the pomegranate peel is a remarkable source of phenolic constituents, including hydrolysable tannins (ellagitannins, i.e., punicalagin isomers, ellagic acid derivatives, and gallic acid), flavonoids, and anthocyanins [15]. Interestingly, the Sripanya pomegranate peel extracts in the present study contained much more phenolic content than previous studies have reported. The total phenolic concentrations of the pomegranate peel from Iran, Turkey, Spain, Southern Tunisia, and South Africa were in a range from 54.84 to $295.5 \mathrm{mg} \mathrm{GAE} / \mathrm{g}$ [6,16-19]. Therefore, Sripanya pomegranate can be suggested as a good souce of natural phenolic compounds. Other than the variety of pomegranate, growing seasons and agricultural practices might also lead to differences in total phenolic content [20].

\subsection{Antioxidant Activities of Sripanya Pomegranate Peel Extracts}

There are several models for analyzing the antioxidant activities of natural compounds. Normally, more than one method is used to analyze and summarize the results because there are various mechanisms inhibiting the oxidation process. However, the present studies employed two different methods to evaluate the free radical scavenging activities of Sripanya pomegranate peel extracts, including the DPPH and ABTS assays. This is because these methods are simple, convenient, and rapid and can be applied to many types of samples.

The $\mathrm{DPPH}^{\bullet}$ scavenging activities of Sripanya pomegranate peel extracts are shown in Figure 3. The E60 extract significantly possessed the most potent $\mathrm{DPPH}^{\bullet}$ scavenging activities with an $\mathrm{IC}_{50}$ of $10.97 \pm 0.97 \mu \mathrm{g} / \mathrm{mL}(p<0.05)$. The ABTS ${ }^{\bullet+}$ scavenging activities, as shown in Figure 4 , were perfectly correlated with the DPPH ${ }^{\bullet}$ scavenging activities. The E60 extract possessed the most potent ABTS $^{\bullet+}$ scavenging activities with an $\mathrm{IC}_{50}$ of $6.48 \pm 0.97 \mu \mathrm{g} / \mathrm{mL}(p<0.05)$. It was noted that Sripanya pomegranate peels extracted using at least $60 \% \mathrm{v} / \mathrm{v}$ of ethanol possessed potent DPPH ${ }^{\bullet}$ and $\mathrm{ABTS}^{\bullet+}$ scavenging activities. These results relate well with their phenolic content. Therefore, phenolic compounds can be suggested as being major components that are responsible for the antioxidant activities of Sripanya pomegranate peel extracts. These results are in accordance with previous 
studies reporting that the presence of phenolic compounds is an important factor in the antioxidant activity of natural products $[15,18]$. Various phenolic compounds have been reported for free radical scavenging activities, including ellagitannins, anthocyanins, gallagylesters, hydroxybenzoic acids, gallotannins, dihydroflavonol, and hydroxycinnamic acids. The proposed mechanism is due to an ability to donate a hydroxyl hydrogen and resonance stabilization from the resulting antioxidant radicals [21]. Additionally, the likely mechanism of the radical scavenging property is the capability of rapidly deactivating reactive oxygen species (ROSs) and reactive nitrogen species (RNSs) via electron transfer [22]. Therefore, Sripanya pomegranate peel extracts, especially the E60 extract, can be suggested as natural antioxidants.

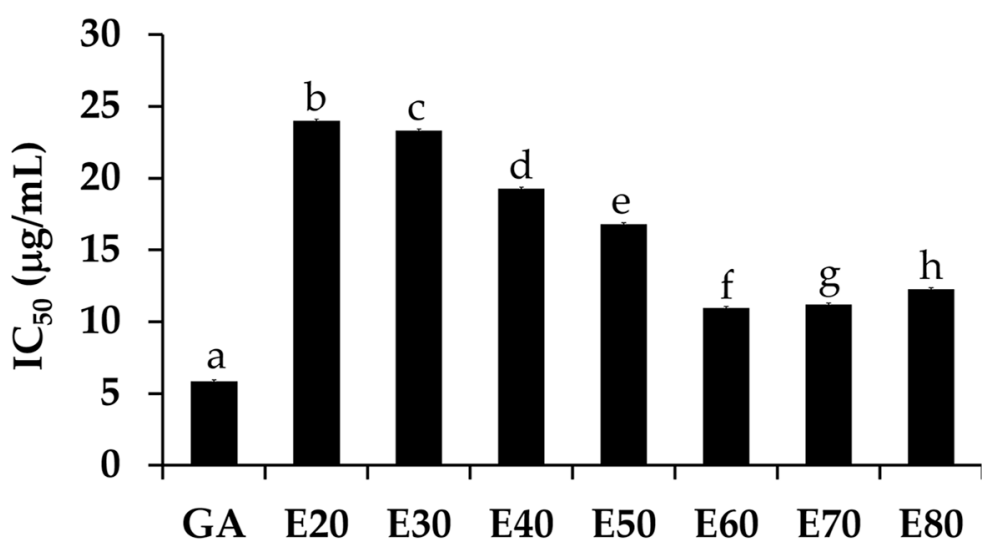

Figure 3. The $\mathrm{IC}_{50}$ values of the $\mathrm{DPPH}^{\bullet}$ scavenging activity of gallic acid (GA) and Sripanya pomegranate peel extracts. E20, E30, E40, E50, E60, E70, and E80 were Sripanya pomegranate peel extracts obtained by maceration in $20 \%, 30 \%, 40 \%, 50 \%, 60 \%, 70 \%$, and $80 \% \mathrm{v} / \mathrm{v}$ of ethanol. Different letters denote statistically significant differences among groups; $(p<0.05)$.

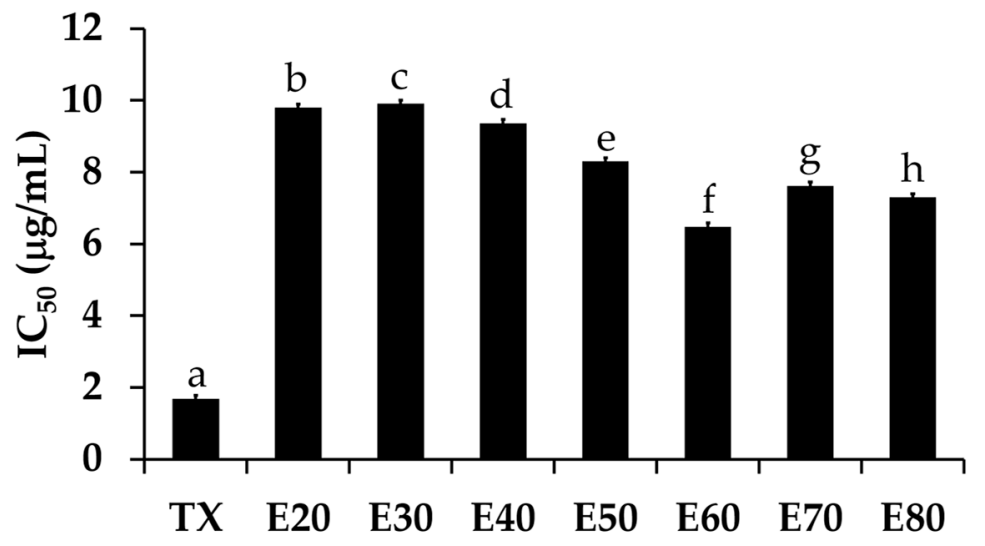

Figure 4. The $\mathrm{IC}_{50}$ values of the $\mathrm{ABTS}^{\bullet+}$ scavenging activity of trolox (TX) and Sripanya pomegranate peel extracts. E20, E30, E40, E50, E60, E70, and E80 were Sripanya pomegranate peel extracts obtained by maceration in $20 \%, 30 \%, 40 \%, 50 \%, 60 \%, 70 \%$, and $80 \% \mathrm{v} / \mathrm{v}$ of ethanol. Different letters denote statistically significant differences among groups; $(p<0.05)$.

\subsection{Tyrosinase Inhibitory Activities of Sripanya Pomegranate Peel Extracts}

Tyrosinase is an enzyme involved in a melanogenesis process, which leads to skin pigmentation and a darker skin color [23]. Inhibition against this enzyme is a major way to whiten the skin. The present study investigated the whitening effect of Sripanya pomegranate peel extracts. The anti-tyrosinase activity of each extract is shown in Figure 5. The E60 extract possessed the most potent anti-tyrosinase activity with an $\mathrm{IC}_{50}$ value of $0.67 \pm 0.98 \mu \mathrm{g} / \mathrm{mL}$. These results relate very well with the total phenolic concentrations of the Sripanya pomegranate peel extracts. Therefore, it can be remarked that phenolic compounds are responsible for the whitening effect of Sripanya pomegranate peels. The likely 
anti-tyrosinase mechanisms of Sripanya pomegranate peel extracts are the chelation of copper atoms and a binding with the tyrosinase hydrophobic binding pocket surrounding the binuclear copper active site [24]. Interestingly, all Sripanya pomegranate peel extracts possessed significantly higher tyrosinase inhibitory activity than kojic acid, a well-known commercial whitening compound, of which the $\mathrm{IC}_{50}$ value was $7.88 \pm 1.00 \mu \mathrm{g} / \mathrm{mL}(p<0.05)$. Therefore, the E60 extract can be suggested for use as a natural whitening compound in further cosmetic product development instead of kojic acid, which reportedly irritates skin [25].

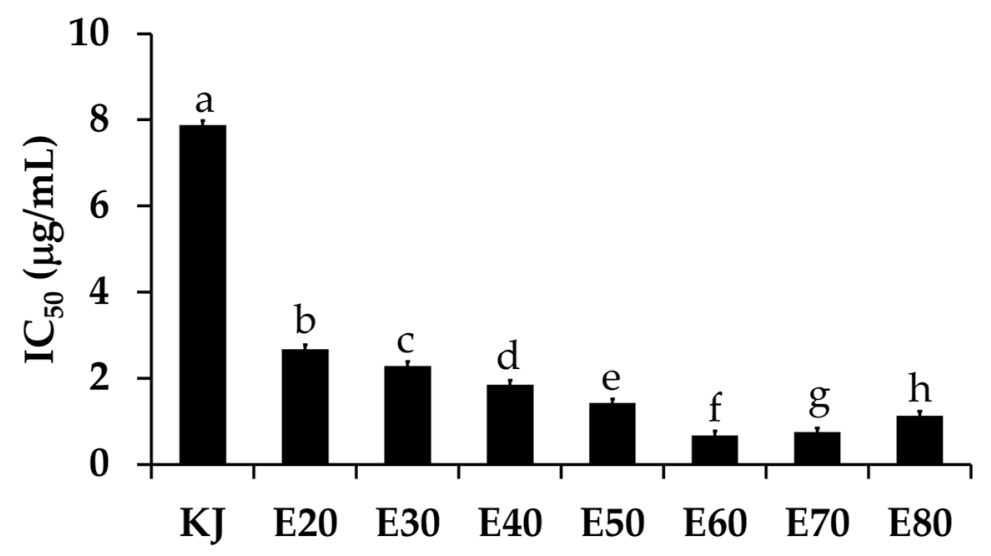

Figure 5. The $\mathrm{IC}_{50}$ values of the tyrosinase inhibitory activity of kojic acid (KJ) and Sripanya pomegranate peel extracts. E20, E30, E40, E50, E60, E70, and E80 were Sripanya pomegranate peel extracts obtained by maceration in $20 \%, 30 \%, 40 \%, 50 \%, 60 \%, 70 \%$, and $80 \% \mathrm{v} / \mathrm{v}$ of ethanol. Different letters denote statistically significant differences among groups; $(p<0.05)$.

\subsection{Chemical Compositions of Sripanya Pomegranate Peel Extracts}

Since the E60 extract possessed the most potent antioxidant and anti-tyrosinase activities, it has been suggested for further use as an active natural compound in cosmetic and cosmeceutical products. However, the standardization of naturally occurring bioactive compounds is very important for the quality control of the products [26]. HPLC was hence used in the present study to investigate the chemical compositions of Sripanya pomegranate peel extracts. Punicalagin and ellagic acid have been reported as major components responsible for various biological activities of pomegranate peel $[16,27,28]$. Therefore, these compounds were used as biological markers for Sripanya pomegranate peel extracts in the present study.

The HPLC chromatogram of the E60 extract is shown in Figure 6. There were several components, but three major components-punicalagin A, punicalagin B, and ellagic acid-were detected at retention times of 16.5, 17.8, and $28.9 \mathrm{~min}$, respectively. Punicalagin A and B were found to be the most abundant components of the E60 extract, with a total concentration of $4.05 \pm 0.26 \% \mathrm{w} / \mathrm{w}$. The results are in accordance with a previous study reporting that punicalagin derivatives are predominant compounds of pomegranate peel [16]. In addition, ellagic acid was another major component, of which the concentration was $0.63 \pm 0.04 \% \mathrm{w} / \mathrm{w}$. These compounds have been suggested for use as active biological markers for the standardization and quality control of Sripanya pomegranate peel extracts. Since punicalagin A, punicalagin B, and ellagic acid have been previously reported as having antioxidant and anti-tyrosinase activities [29], it can be said that these compouds are responsible for the potent biological activities of the E60 extract. Ellagic acid has been reported to bind with copper with high affinity at the active site of tyrosinase and hence inhibits the tyrosinase function in melanogenesis $[6,7,30]$. Additionally, ellagic acid has been found to suppress the UV-induced skin pigmentation of guinea pigs [7]. On the other hand, both punicalagins have been reported as showing dose-dependently decreased tyrosinase activity via a downregulation of microphthalmia-associated transcription factor (MITF) and tyrosinase expression [29]. Therefore, both punicalagins and ellagic acid were considered in this study as major bioactive components of Sripanya pomegranate peel extracts, 
especially the E60 extract. Furthermore, these compounds can be suggested for use as biological markers for quality control or quantitative determination of Sripanya pomegranate peel extracts in future experiments.

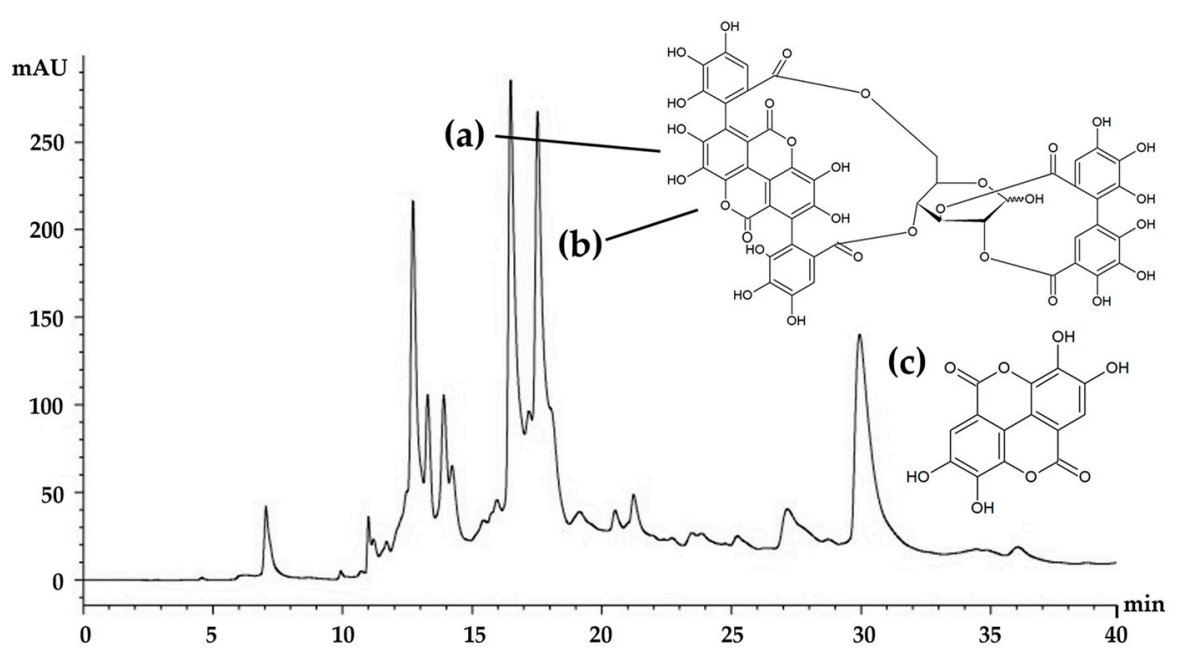

Figure 6. HPLC chromatogram of the E60 extract, which was Sripanya pomegranate peel extract obtained by maceration in $60 \% \mathrm{v} / \mathrm{v}$ of ethanol. (a) and (b) are punicalagin derivatives, whereas (c) is ellagic acid.

\subsection{Irritation on the Chorioallantoic Membrane of the Sripanya Pomegranate Peel Extracts}

Since irritation is a major consideration for cosmetic and cosmeceutical product development, the E60 extract, which has been suggested for use as an antioxidant and a whitening agent, was investigated for irritation effect in the hen's egg test-chorioallantoic membrane (HET-CAM) assay. The CAM was examined for vascular damage, including vascular hemorrhages, vascular lysis, and vascular coagulation, after being exposed to the E60 solution at concentrations ranging from $10 \mu \mathrm{g} / \mathrm{mL}$ to $1 \mathrm{mg} / \mathrm{mL}$. The IS scores as shown in Table 1 show that $1 \% \mathrm{w} / \mathrm{v}$ SLS (a positive control) induced severe irritation, whereas $0.9 \% \mathrm{w} / \mathrm{v} \mathrm{NaCl}$ (a negative control) induced no irritation. Moreover, SLS induces vascular lysis as shown in Figure 7. On the other hand, the E60 extract did not induce any irritation at any concentrations tested, as shown in Figure 8. Therefore, we suggest that the E60 extract is a safe natural compound for topical application.

Table 1. Irritation score and irritation assessment of Sripanya pomegranate peel extract by the HET-CAM method.

\begin{tabular}{lcc}
\hline \multicolumn{1}{c}{ Samples } & Irritation Score & Irritation Assessment \\
\hline Positive control $(1 \% \mathrm{w} / \mathrm{v} \mathrm{SLS})$ & $9.12 \pm 0.15$ & Severe irritation \\
Negative control $(0.9 \% \mathrm{w} / \mathrm{v} \mathrm{NaCl})$ & $0.00 \pm 0.00$ & No irritation \\
E60 $(10 \mu \mathrm{g} / \mathrm{mL})$ & $0.00 \pm 0.00$ & No irritation \\
E60 $(50 \mu \mathrm{g} / \mathrm{mL})$ & $0.00 \pm 0.00$ & No irritation \\
$\mathrm{E} 60(100 \mu \mathrm{g} / \mathrm{mL})$ & $0.00 \pm 0.00$ & No irritation \\
$\mathrm{E} 60(250 \mu \mathrm{g} / \mathrm{mL})$ & $0.00 \pm 0.00$ & No irritation \\
$\mathrm{E} 60(500 \mu \mathrm{g} / \mathrm{mL})$ & $0.00 \pm 0.00$ & No irritation \\
$\mathrm{E} 60(1 \mathrm{mg} / \mathrm{mL})$ & $0.00 \pm 0.00$ & No irritation \\
E60 $(5 \mathrm{mg} / \mathrm{mL})$ & $0.00 \pm 0.00$ & No irritation \\
\hline
\end{tabular}

The data are expressed as means \pm SD; duplicates; $n=2$; E60 was Sripanya pomegranate peel extract obtained by maceration in $60 \% \mathrm{v} / \mathrm{v}$ of ethanol. 


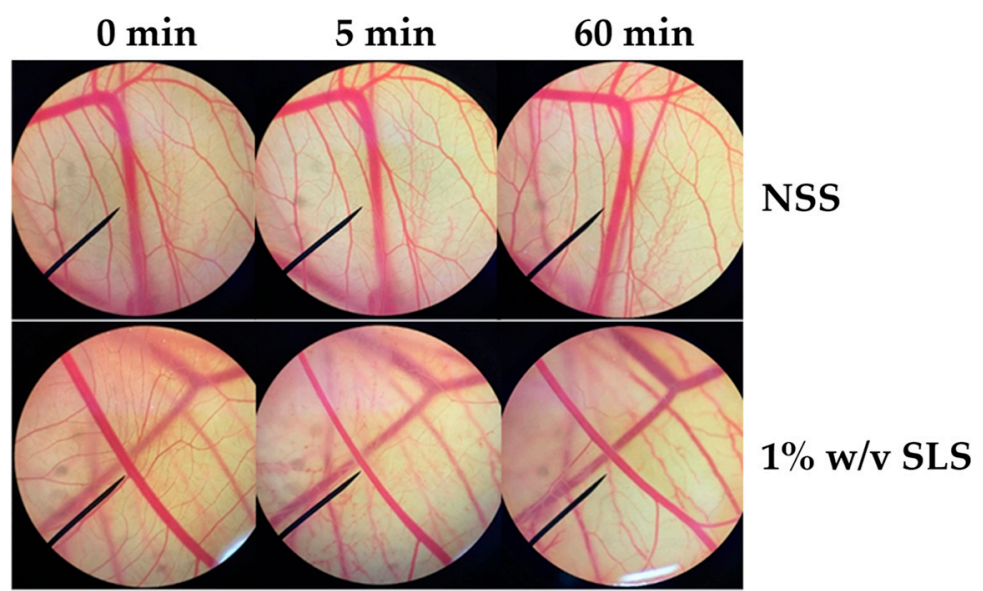

Figure 7. Photographs of the chorioallantoic membrane before and after exposure to $0.9 \% \mathrm{w} / \mathrm{v}$ sodium chloride $(\mathrm{NaCl})$ and $1 \% \mathrm{w} / \mathrm{v}$ sodium lauryl sulfate (SLS).

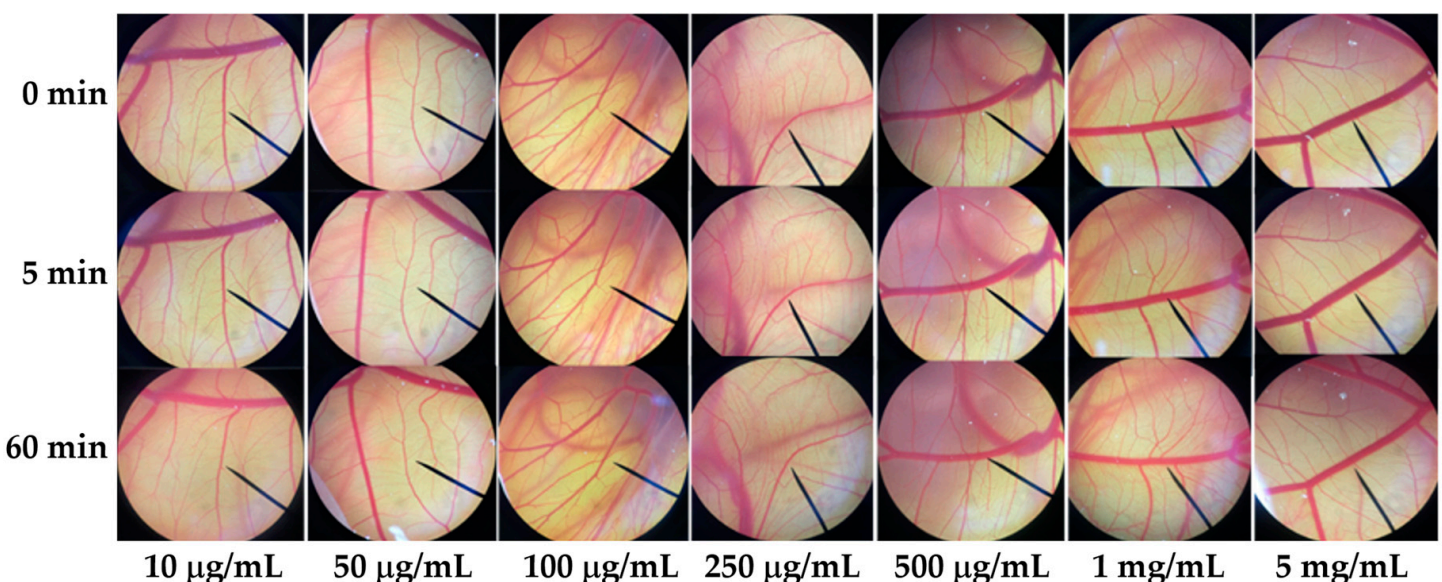

Figure 8. Photographs of the chorioallantoic membrane before and after exposure to the Sripanya pomegranate peel extract obtained by maceration in $60 \% \mathrm{v} / \mathrm{v}$ of ethanol (E60).

\section{Conclusions}

The present study revealed the most effective concentration of ethanol for extracting bioactive compounds from Sripanya pomegranate peel. E60, which was Sripanya pomegranate peel extracts by maceration in $60 \% \mathrm{v} / \mathrm{v}$ of ethanol, had the highest yield $(66.23 \pm 0.38 \% \mathrm{w} / \mathrm{w})$, the most abundant total phenolic content ( $490.42 \pm 7.20 \mu \mathrm{g} \mathrm{GAE} / \mathrm{g})$, and the most potent biological activities, including antioxidant and anti-tyrosinase activity. The $\mathrm{IC}_{50}$ values on $\mathrm{DPPH}^{\bullet}$ and $\mathrm{ABTS}^{\bullet+}$ inhibition were $10.97 \pm 0.97$ and $6.48 \pm 0.06 \mu \mathrm{g} / \mathrm{mL}$, respectively. Furthermore, the E60 extract possessed strong tyrosinase inhibitory activity with an $\mathrm{IC}_{50}$ value of $0.10 \pm 0.13 \mu \mathrm{g} / \mathrm{mL}$, which is more potent than kojic acid $\left(\mathrm{IC}_{50}=7.88 \pm 1.00 \mu \mathrm{g} / \mathrm{mL}\right)$, a commercial whitening agent. Moreover, phenolic compounds were found to be responsible for both antioxidant and anti-tyrosinase activity. Punicalagin and ellagic acid were found to be the major constituents of the E60 extract, with amounts of $4.05 \pm 0.26 \% \mathrm{w} / \mathrm{w}$ and $0.63 \pm 0.04 \% \mathrm{w} / \mathrm{w}$, respectively. The E60 extract can safely be developed as a topical product since it showed no irritation on a CAM. Therefore, the E60 extract is promising as an active agent for pharmaceutical and cosmeceutical purposes. Additionally, further biological activities, including the anti-inflammatory and anti-aging activities of the E60 extract, can be considered for a variety of other applications in the field of cosmetics.

Author Contributions: Investigation: N.L., C.S., and W.C.; formal analysis: N.L., C.S., S.E., B.S., and W.C.; writing - original draft, reviewing, and editing: N.L. and W.C.; funding acquisition: N.L. and B.S.; supervision and project administration: J.S. and B.S. All authors have read and agreed to the published version of the manuscript. 
Funding: This research was funded by the National Research Council of Thailand (grant number P000019871, a grant for graduate student 2560), the Graduate School at Chiang Mai University, Thailand, and the Faculty of Pharmacy at Chiang Mai University.

Conflicts of Interest: The authors declare that there is no conflict of interest.

\section{References}

1. Rahimi, H.R.; Arastoob, M.; Ostada, S.N. A comprehensive review of Punica granatum (Pomegranate) properties in toxicological, pharmacological, cellular and molecular biology researches. Iran. J. Pharm. Res. 2012, 11, 385-400. [PubMed]

2. Mousavinejad, G.; Emam-Djomeh, Z.; Rezaei, K.; Khodaparast, M.H.H. Identification and quantification of phenolic compounds and their effects on antioxidant activity in pomegranate juices of eight Iranian cultivars. Food Chem. 2009, 115, 1274-1278. [CrossRef]

3. Zhao, X.; Yuan, Z.; Fang, Y.; Yin, Y.; Feng, L. Characterization and evaluation of major anthocyanins in pomegranate (Punica granatum L.) peel of different cultivars and their development phases. Eur. Food Res. Technol. 2013, 236, 109-117. [CrossRef]

4. Aslam, M.N.; Lansky, E.P.; Varani, J. Pomegranate as a cosmeceuticals source: Pomegranate fractions promote proliferation and procollagen synthesis and inhibit matrix metalloproteinase-1 production in human skin cells. J. Ethnopharmacol. 2006, 103, 311-318. [CrossRef]

5. World Health Organization. WHO Monographs on Selected Medicinal Plants, 1st ed.; World Health Organization: Geneva, Switzerland, 2009; pp. 117-126.

6. Fawole, O.A.; Makunga, N.P.; Opara, U.L. Antibacterial, antioxidant and tyrosinase-inhibition activities of pomegranate fruit peel methanolic extract. Bmc Complem. Altern. Med. 2012, 12,1-11. [CrossRef]

7. Yoshimura, M.; Watanabe, Y.; Kasai, K.; Yamakoshi, J.; Koga, T. Inhibitory effect of an ellagic acid-rich pomegranate extract on tyrosinase activity and ultraviolet-induced pigmentation. Biosci. Biotechnol. Biochem. 2005, 69, 2368-2373. [CrossRef]

8. Li, H.B.; Cheng, K.W.; Wong, C.C.; Fan, K.W.; Chen, F.; Jiang, Y. Evaluation of antioxidant capacity and total phenolic content of different fractions of selected microalgae. Food Chem. 2007, 102, 771-776. [CrossRef]

9. Brem, B.; Seger, C.; Pacher, T.; Hartl, M.; Hadacek, F.; Hofer, O.; Vajrodaya, S.; Greger, H. Antioxidant dehydrotocopherols as a new chemical character of Stemona species. Phytochemistry 2004, 65, 2719-2729. [CrossRef]

10. Tachakittirungrod, S.; Okonogi, S.; Chowwanapoonpohn, S. Study on antioxidant activity of certain plants in Thailand: Mechanism of antioxidant action of guava leaf extract. Food Chem. 2007, 103, 381-388. [CrossRef]

11. Saeio, K.; Chaiyana, W.; Okonogi, S. Antityrosinase and antioxidant activities of essential oils of edible Thai plants. Drug Discov. Ther. 2011, 5, 144-149. [CrossRef] [PubMed]

12. Li, J.; He, X.; Li, M.; Zhao, W.; Liu, L.; Kong, X. Chemical fingerprint and quantitative analysis for quality control of polyphenols extracted from pomegranate peel by HPLC. Food Chem. 2005, 176, 7-11. [CrossRef]

13. Steiling, W.; Bracher, M.; Courtellemont, P.; De Silva, O. The HET-CAM, a useful in vitro assay for assessing the eye irritation properties of cosmetic formulations and ingredients. Toxicol. Vitr. 1999, 13, 375-384. [CrossRef]

14. Chaiyana, W.; Punyoyai, C.; Somwongin, S.; Leelapornpisid, P.; Ingkaninan, K.; Waranuch, N.; Srivilai, J.; Thitipramote, N.; Wisuitiprot, W.; Schuster, R.; et al. Inhibition of 5-Reductase, IL-6 secretion, and oxidation process of Equisetum debile Roxb. ex Vaucher extract as functional food and nutraceuticals ingredients. Nutrients 2017, 9, 1105. [CrossRef] [PubMed]

15. Ismail, T.; Sestili, P.; Akhtar, S. Pomegranate peel and fruit extracts: A review of potential anti-inflammatory. J. Ethnopharmacol. 2012, 143, 397-405. [CrossRef] [PubMed]

16. Çam, M.; Yaşar, H. Pressurised water extraction of polyphenols from pomegranate peels. Food Chem. 2010, 123, 878-885.

17. Yasoubi, P.; Barzegar, M.; Sahari, M.A.; Azizi, M.H. Total phenolic contents and antioxidant activity of pomegranate (Punica granatum L.) peel extracts. J. Agri. Sci. Technol. 2007, 9, 35-42.

18. Viuda-Martos, M.; Pérez-Álvarez, J.; Sendra, E.; Fernández-López, J. In vitro antioxidant properties of pomegranate (Punica Granatum) peel powder extract obtained as coproduct in the juice extraction process. J. Food Process. Preserv. 2013, 37, 772-776. [CrossRef] 
19. Elfalleh, W.; Hannachi, H.; Tlili, N.; Yahia, Y.; Nasri, N.; Ferchichi, A. Total phenolic contents and antioxidant activities of pomegranate peel, seed, leaf and flower. J. Med. Plants Res. 2012, 6, 4724-4730. [CrossRef]

20. Legua, P.; Melgarejo, P.; Abdelmajid, H.; Martínez, J.J.; Martínez, R.; Ilham, H.; Hafida, H.; Hernández, F. Total phenols and antioxidant capacity in 10 Moroccan pomegranate varieties. J. Food Sci. 2012, 71, 115-120. [CrossRef]

21. Nimse, S.B.; Pal, D. Free radicals, natural antioxidants, and their reaction mechanisms. Rsc Adv. 2015, 5, 27986-28006. [CrossRef]

22. Marino, T.; Galano, A.; Russo, N. Radical scavenging ability of gallic acid toward $\mathrm{OH}$ and OOH radicals. Reaction mechanism and rate constants from the density functional theory. J. Phys. Chem. B 2014, 118, 10380-10389. [CrossRef] [PubMed]

23. Pillaiyar, T.; Manickam, M.; Namasivayam, V. Skin whitening agents: Medicinal chemistry perspective of tyrosinase inhibitors. J. Enzym. Inhib. Med. Chem. 2017, 32, 403-425. [CrossRef] [PubMed]

24. Nithitanakool, S.; Pithayanukul, P.; Bavovada, R.; Saparpakorn, P. Molecular docking studies and anti-tyrosinase activity of Thai mango seed kernel extract. Molecules 2009, 14, 257-265. [CrossRef]

25. Rendon, M.I.; Gaviria, J.I. Review of skin-lightening agents. Derm. Surg. 2005, 31, 886-890. [CrossRef] [PubMed]

26. Lazarowych, N.J.; Pekos, P. Use of fingerprinting and marker compounds for identification and standardization of botanical drugs: Strategies for applying pharmaceutical HPLC analysis to herbal products. Drug Inf. J. 1998, 32, 497-512. [CrossRef]

27. Qua, W.; Breksa, A.P., III; Pan, Z.; Ma, H. Quantitative determination of major polyphenol constituents in pomegranate products. Food Chem. 2012, 132, 1585-1591. [CrossRef]

28. Oudane, B.; Boudemagh, D.; Bounekhel, M.; Sobhi, W.; Vidal, M.; Broussy, S. Isolation, characterization, antioxidant activity, and proteinprecipitating capacity of the hydrolyzable tannin punicalagin from pomegranate yellow peel (Punica granatum). J. Mol. Struct. 2018, 1156, 390-396. [CrossRef]

29. Shin, J.S.; Cho, J.H.; Lee, H.; Jeong, H.S.; Kim, M.K.; Yun, H.Y.; Kwon, N.S.; Kim, D.S. Dual hypopigmentary effects of punicalagin via the ERK and Akt pathways. Biomed. Pharm. 2017, 92, 122-127. [CrossRef]

30. Akhtar, S.; Ismail, T.; Fraternale, D.; Sestili, P. Pomegranate peel and peel extracts: Chemistry and food features. J. Food Chem. 2015, 174, 417-425. [CrossRef] 